\title{
Interleukin-I antagonists in the treatment of autoinflammatory syndromes, including cryopyrin-associated periodic syndrome
}

This article was published in the following Dove Press journal:

Open Access Rheumatology: Research and Reviews

18 January 2011

Number of times this article has been viewed

\section{Pierre Quartier \\ Unité d'Immunologie-Hématologie et Rhumatologie pédiatriques, Hôpital Necker-Enfants Malades, Assistance Publique-Hôpitaux de Paris, Paris, France}

Correspondence: Pierre Quartier Université Paris-Descartes, INSERM U768, Centre de Référence Maladies Rares, Arthrites Juvéniles, Unité d'Immunologie-Hématologie et Rhumatologie pédiatriques, Hôpital Necker-Enfants Malades, Assistance Publique-Hôpitaux de Paris, Paris, France Tél +33 I44 494828

Fax +33 I44 495070

Email quartier@necker.fr

\begin{abstract}
Cryopyrin-associated periodic syndrome (CAPS) include a group of rare autoinflammatory disorders, the spectrum of which ranges from the mildest form, ie, familial cold autoinflammatory syndrome to more severe phenotypes, ie, Muckle-Wells syndrome, and chronic infantile neurological cutaneous and articular syndrome, also known as neonatal-onset multisystem inflammatory disease. Three interleukin (IL)-1 antagonists have been tested in adults and children with CAPS, ie, anakinra, a recombinant homolog of the human IL-1 receptor antagonist; rilonacept, a fusion protein comprising the extracellular domains of IL-1 receptor I and the IL-1 adaptor protein, IL-1RAcP, attached to a human immunoglobulin G molecule; and canakinumab, the anti-IL-1 $\beta$ monoclonal antibody. Following rapid clinical development rilonacept and canakinumab were approved by both the US Food and Drug Administration and the European Medicines Agency for use in adults and children. This review describes how the study of CAPS has helped us to understand better the way the innate immune system works, the pathogenesis of autoinflammatory syndromes, and the key role of IL-1. It also reviews the effects of IL-1 blockade in CAPS and other disorders, in particular systemic juvenile idiopathic arthritis, adult-onset Still's disease, and gout. Finally, this review covers some issues addressed by very recent and ongoing work regarding treatment indications, from orphan diseases to common disorders, continuous versus intermittent treatment, the pharmacokinetics, pharmacodynamics, and optimal dosages of the different drugs, as well as the need for Phase IV trials, exhaustive registries, and long-term follow-up of several patient cohorts.
\end{abstract}

Keywords: inflammation, interleukin-1, cytokines, treatment

\section{Introduction}

The fully human anti-interleukin (IL)-1 $\beta$ monoclonal antibody, canakinumab, was approved in 2010 by both the US Food and Drug Administration and the European Medicines Agency for use in adults and children with cryopyrin-associated periodic syndrome (CAPS). When approval was requested, fewer than 1000 patients had been receiving canakinumab, some for CAPS and some for other conditions. In addition, two other IL-1 inhibitors had also been tested in the same indications, ie, anakinra, a recombinant homolog of the human IL-1 receptor antagonist, and rilonacept, a fusion protein comprising the extracellular domains of the IL-1 receptor I and the IL-1 adaptor protein, IL-1RAcP, attached to a human immunoglobulin G molecule. Both agencies have also approved the use of rilonacept in patients with CAPS.

This review aims to show the rationale for this extremely rapid development and to discuss some important questions which are still unresolved. Firstly, this paper describes how studying CAPS has been important in order to understand better the way 
in which the innate immune system works, the pathogenesis of autoinflammatory syndromes, and the key role of IL-1. Secondly, the effect of IL-1 blockade in CAPS and other disorders is discussed. Some issues, addressed by very recent and ongoing work and projects, are also mentioned.

\section{Methods}

The PubMed database was searched for original papers published in English from 2002 until December 21, 2010 containing in their title or abstract the search terms "anti-IL-1 treatment", "anti-IL-1 therapy", "anakinra", "rilonacept", "canakinumab", "familial cold urticaria", "autoinflammatory syndrome", "Muckle-Wells", "chronic infantile neurological cutaneous and articular syndrome", "neonatal-onset multisystem inflammatory disease", "systemic juvenile arthritis", "Still's disease", and "gout". The review also included abstracts from the European League against Rheumatism and American College of Rheumatism meetings from 2004 onwards. The author also based this review on personal clinical experience.

\section{Cryopyrin-associated periodic syndrome}

CAPS includes a group of rare autoinflammatory disorders, the spectrum of which ranges from its mildest form, ie, familial cold autoinflammatory syndrome, to more severe phenotypes, ie, Muckle-Wells syndrome and chronic infantile neurological cutaneous and articular syndrome (CINCA), also known as neonatal-onset multisystem inflammatory disease (NOMID). ${ }^{1-5}$

Patients with familial cold autoinflammatory syndrome are typically only symptomatic upon exposure to cold. Exposure to cold induces an urticarial rash, fever, chills, joint pain, conjunctivitis, and headaches. In these patients, the functional consequences of the disease are usually mild, and amyloidosis is exceptional. ${ }^{2}$ Similar symptoms are found in Muckle-Wells syndrome, usually starting in childhood or the teenage years. Triggering by cold can be present, but disease flares may also develop in the absence of any trigger and last one or more days. Muckle-Wells syndrome is also associated with progressive sensorineural hearing loss in at least $75 \%$ of patients whose symptoms start before adulthood with, in some cases, visual problems and secondary amyloid A amyloidosis, leading to renal impairment. ${ }^{1,6}$

CINCA is characterized by a very early onset, usually within the first days or weeks of life, sometimes before birth. ${ }^{3-5}$ Affected individuals present with some dysmorphic features (Figure 1). An urticarial-like rash is usually present at this early stage (Figure 2), but may vary in intensity during
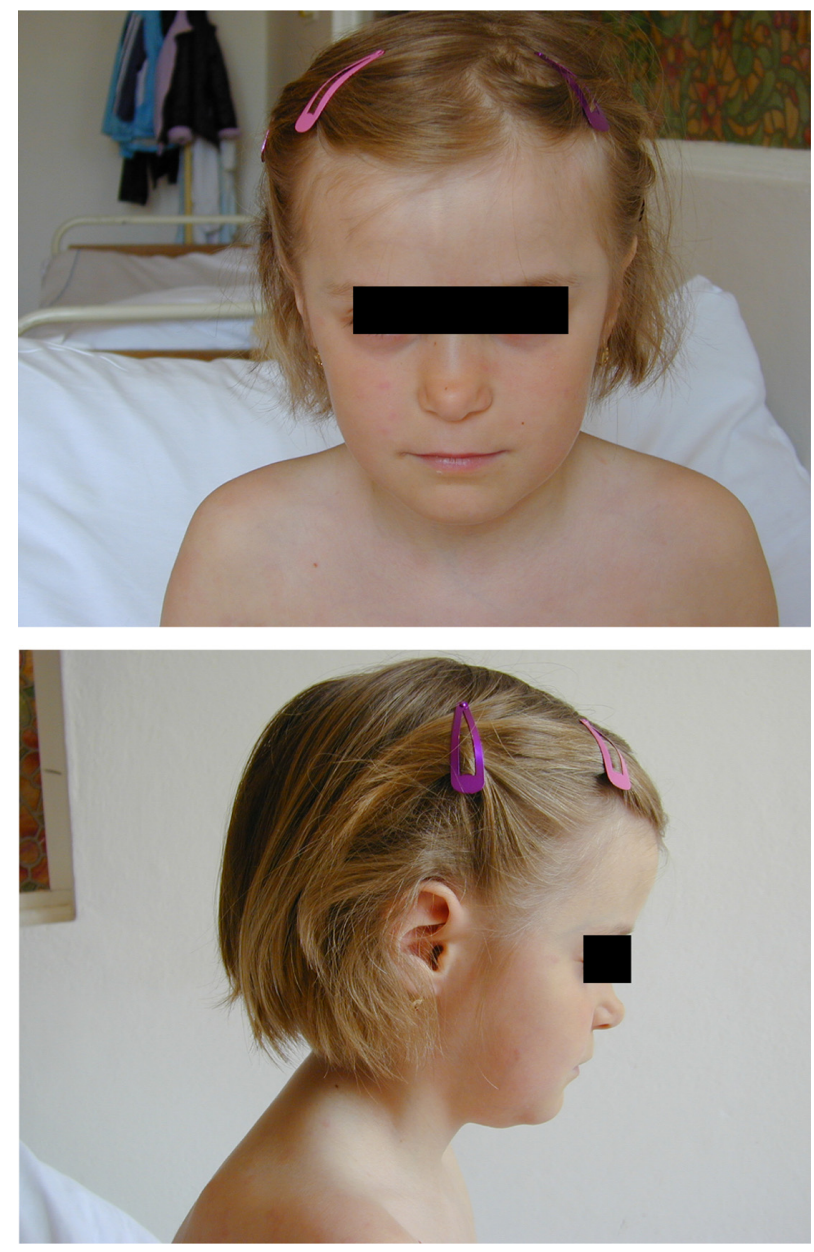

Figure I

the day, and from one day to another. Fever spikes, joint pain, myalgia, abdominal pain, and headaches become increasingly intense. Patients suffer from progressive chronic meningitis, worsening of visual problems (papilledema, sight loss) and sensorineural hearing loss. Failure to thrive, pubertal delay, and mental retardation may be present in teenage patients. Amyloid A amyloidosis develops in these patients and may include renal and thyroid involvement, sometimes as early as childhood. In a subset of CINCA patients, overgrowth of the patella and epiphyses of long bones can develop, resulting in gross deformity of the joints (Figure 3 ). In addition to a permanently increased erythrocyte sedimentation rate, C-reactive protein, and serum amyloid, CINCA patients typically show an increased number of neutrophils in their blood count, cerebrospinal fluid (together with hyperproteinorachia), and every affected organ, except for hypertrophic bone and cartilage.

Initially described as three distinct disorders, the recognition of familial cold autoinflammatory syndrome (MIM 120100), Muckle-Wells syndrome (MIM 191900), and 


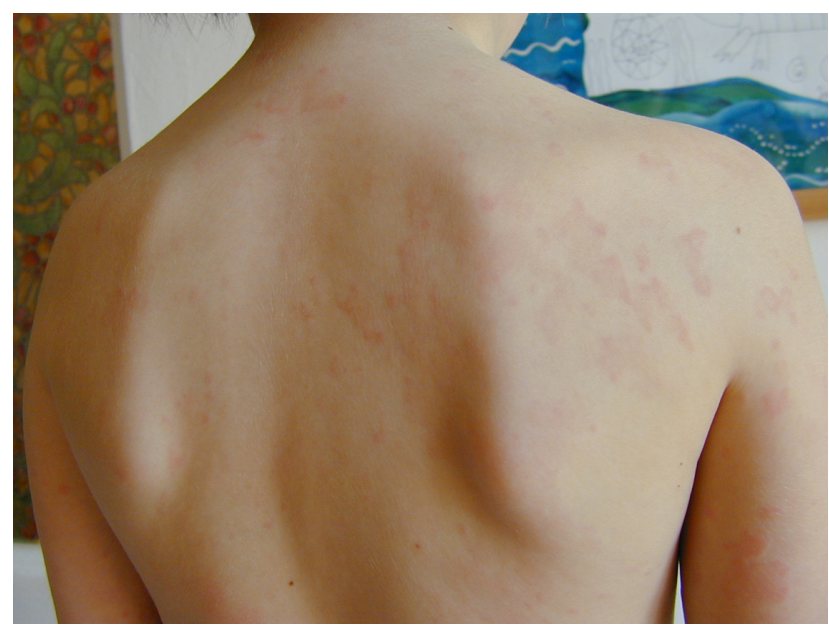

Figure 2

CINCA/NOMID (MIM607115) as the spectrum of a single disorder arose following linkage studies, which mapped both Muckle-Wells syndrome and familial cold autoinflammatory syndrome to chromosome $11 \mathrm{q} 44,{ }^{6,7}$ and identification of the mutated gene as CIASI, the gene encoding NLRP3, which plays a critical role in IL-1 processing..$^{8-12}$

Familial cold autoinflammatory syndrome and MuckleWells syndrome usually present as an autosomal dominant inherited disorder. In contrast, most CINCA patients show neomutations. There is a correlation in the genotype/phenotype, with some mutations systematically associated with milder forms of the disease (familial cold autoinflammatory syndrome, Muckle-Wells syndrome) while other mutations are only found in severe Muckle-Wells syndrome or CINCA patients. ${ }^{13}$ In some cases, no NLRP3 mutation is found. ${ }^{9,10}$

\section{Innate immune system and IL-I $\beta$}

The study of CAPS has played a major role in clarifying the essential function of key elements of the innate immune system, in particular the cytokine IL-1 $\beta .{ }^{10,11}$ This has enabled the successful development of new therapeutic strategies in a great variety of diseases.

The innate immune system is the first line of defense against danger signals, including life-threatening pathogens. ${ }^{11,12}$ These are recognized by pattern-recognition receptors which activate various signaling cascades leading to inflammatory responses. There are two main types of pattern-recognition receptors, ie, membrane-bound Toll-like receptors and soluble proteins found in the cytoplasm, of which two families have been described, ie, NOD-like receptors and RIG-like helicases.

Among several members of the NOD-like receptor family, the largest family, known as NALP, is characterized

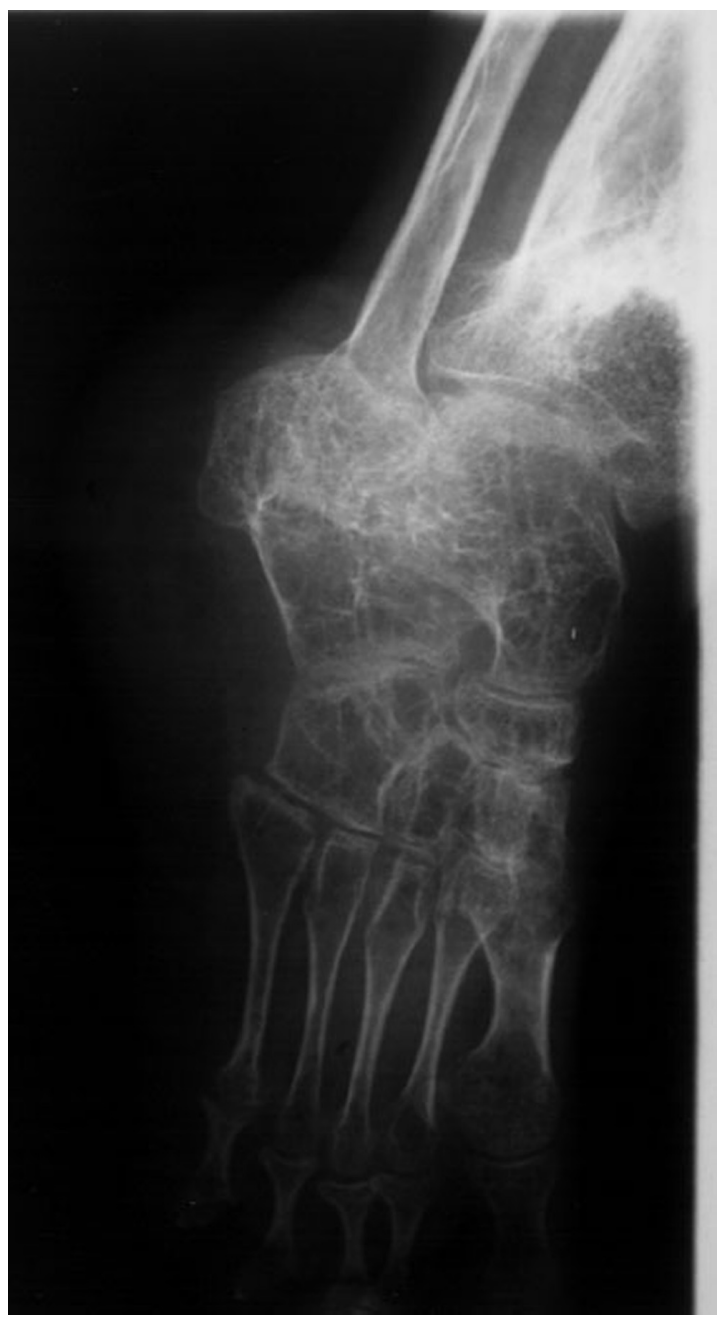

Figure 3

as having a pyrin domain at the N-terminal end. NALP1, NALP2, and NALP3 are the central scaffold of a protein complex known as the inflammasome. The inflammasome is involved in activating caspase- 1 , a protease which cleaves pro-IL- $1 \beta$ to IL- $1 \beta .{ }^{11,12} \mathrm{IL}-1 \beta$ is a highly active proinflammatory cytokine. Its synthesis and release are tightly regulated, and the activity of the released IL-1 $\beta$ is further regulated by IL-1Ra, the endogenous antagonist of the IL-1 receptor, IL-1R1. ${ }^{14}$

NALP1, NALP2, and NALP3 have been implicated in various autoinflammatory syndromes, and may be involved in many diseases now recognized as having an important inflammatory component. ${ }^{11,12}$

In addition to CAPS syndrome, the link between the mutated gene and overproduction of IL-1 $\beta$ has been elucidated for some other autoinflammatory syndromes, such as familial Mediterranean fever ${ }^{15}$ or deficiency in the IL-1 receptor antagonist (DIRA). ${ }^{16}$ Familial Mediterranean fever 
is associated with gain-of-function mutations in the gene encoding the protein pyrin/marenostrin, resulting in overproduction of IL-1 $\beta$. It is thought that pyrin may prevent formation of a functional NALP3 inflammasome complex by binding to apoptosis-associated speck-like protein with CARD domain, a key protein of the NALP3 inflammasome. In addition, pyrin binds to caspase- 1 and inhibits the ability of caspase- 1 to cleave pro-IL-1 $\beta$. In DIRA, mutations or deletions result in an IL-1 receptor antagonist deficiency.

However, for other autoinflammatory disorders, such as tumor necrosis factor- $\alpha$ receptor-associated periodic syndrome or mevalonate kinase deficiency/hyperimmunoglobulin D syndrome, there is no clear understanding of the link between the mutations found in these patients and the fact that IL-1 $\beta$ blockade is often effective in treating the symptoms of the disease. In other diseases, such as systemic juvenile idiopathic arthritis and adult onset Still's disease, there is no evidence of an underlying mutation and no clear understanding of the pathogenesis of the disease, but most patients respond to IL-1 blockade.

\section{Efficacy and safety of IL-I inhibition in CAPS}

The three anti-IL-1 $\beta$ agents that have been studied in the treatment of CAPS and other inflammatory disorders are: anakinra, a recombinant nonglycosylated homolog of the human IL-1 receptor antagonist, which competitively inhibits the binding of IL- $1 \alpha$ and IL- $1 \beta$ to the IL- 1 receptor; rilonacept, a fusion protein comprising the extracellular domains of IL-1 receptor I and the IL-1 adaptor protein, IL-1RAcP, attached to a human IgG molecule; and canakinumab. These three drugs are almost exclusively administered subcutaneously. However, intravenous infusions are performed in some cases, in particular, in a few patients taking part in a Phase II trial with canakinumab.

The first demonstration of the efficacy of IL-1 inhibition in CAPS came in 2003 through the treatment of two patients by anakinra with Muckle-Wells syndrome who had high serum levels of amyloid A. A dramatic reduction in inflammatory symptomatology was observed within hours of the first injection of anakinra, accompanied by normalization of serum amyloid A levels within three days. ${ }^{17}$

In another paper, published in 2004, four patients with familial cold autoinflammatory syndrome patients and three healthy control patients were exposed to cold challenge with and without anakinra administered at 24 hours and one hour beforehand. Anakinra prevented the development of signs and symptoms associated with cold challenge in patients with familial cold autoinflammatory syndrome, who remained symptom-free for 24-48 hours after the second dose. ${ }^{18}$ In addition, anakinra prevented an increase in the white cell count and IL-6 associated with cold challenge. The clinical efficacy of anakinra and its effect on C-reactive protein and serum amyloid A were confirmed in another open-label study, which included eight patients with familial cold autoinflammatory syndrome who received anakinra for 28 days and were exposed to cold challenges before, during, and after treatment. ${ }^{19}$

A publication from 2006 reported on the efficacy at six months of anakinra treatment in 18 patients with CINCA. Scoring on the specific clinical score constructed for the purpose of the trial, was significantly decreased at three and six months as compared with baseline measurements. ${ }^{20}$

Other reports have also shown that anakinra is effective in CAPS patients. ${ }^{21-27}$ Several of these patients were followed up for more than three years. ${ }^{26,27}$ In almost all cases, fever, chills, urticaria-like rash, arthralgia, myalgia, and headaches resolved within a few hours or days. Acutephase reactants normalized quickly or reached near normal values. Anakinra treatment was also associated with marked improvement of growth velocity and pubertal development in teenage patients. Quality of life also improved. ${ }^{27}$ Some reports showed improvement in hearing as well as amyloid-related proteinuria in patients with Muckle-Wells syndrome or CINCA. However, hearing loss, which probably results from early cochlear necrosis in most cases, as well as amyloidosis, does not recover in the majority of patients. In patients with CINCA, abnormalities in the cerebrospinal fluid tended to resolve or improve over time. Our team and others have shown that patients with CINCA have brain magnetic resonance imaging abnormalities, consisting of abnormal enhancement of small vessels of the basal ganglia after contrast injection and periventricular white matter lesions in fluid-attenuated inversion recovery sequences, as well as ventriculomegaly in a few cases. Our team has also reported that such abnormalities tend to resolve or improve with anakinra treatment. ${ }^{26}$

Of note, very little is known regarding the pharmacokinetics and pharmacodynamics of anakinra in children. Our team showed that young, low-weight CINCA children required, in some cases, up to $4-5 \mathrm{mg} / \mathrm{kg}$ every 12 hours, ${ }^{26}$ whilst adult patients with familial cold autoinflammatory syndrome or Muckle-Wells syndrome were controlled by daily anakinra doses ranging from $0.5 \mathrm{mg} / \mathrm{kg}$ to $1 \mathrm{mg} / \mathrm{kg}$. 
In these trials, injection site reactions were present in up to $50 \%$ of patients (and even more had pain upon injection), but this was mild in most cases, and tended to decrease over time. Few serious adverse events were documented. However, an increased risk of bacterial infection is possible.

Rilonacept has also been tested in CAPS patients, with two major publications concerning patients with familial cold autoinflammatory syndrome or Muckle-Wells syndrome in 2008..$^{28,29}$ One open-label Phase II trial in patients with familial cold autoinflammatory syndrome showed that all patients responded within 24 hours, and disease flare occurred 10-28 days later. ${ }^{28}$ In one Phase III trial of 47 patients with familial cold autoinflammatory syndrome $(\mathrm{n}=44)$ or Muckle-Wells syndrome $(\mathrm{n}=3)$, treatment comprised weekly rilonacept injections at a dose of $160 \mathrm{mg}$ in a six-week, randomized, double-blind, placebo-controlled phase, followed by nine weeks of single-blind treatment with rilonacept, followed by a nine-week, randomized, double-blind, placebo-controlled withdrawal phase. Significant differences were recorded between rilonacept and placebo during both the first and third phases of the trial. Injection site reactions occurred in $48 \%$ of patients receiving rilonacept and in $13 \%$ of patients receiving placebo in the first part, whereas this figure was 36\% versus $13 \%$ in the third part. ${ }^{29}$ Initially, only one serious adverse event was reported and was not considered to be related to treatment. Later, a 70-year-old patient died from a pneumococcal infection.

Canakinumab is the only IL-1 inhibitor that is specific to IL-1 $\beta$. In a study in which canakinumab was administered to a healthy control panel and to CAPS patients, the resulting formation of IL-1-antibody complexes allowed the detection of IL-1 $\beta$ produced in vivo. A two-compartment mathematical model was generated that predicted a constitutive production rate of $6 \mathrm{ng} /$ day of IL-1 $\beta$ in healthy subjects. In contrast, CAPS patients produced a mean of $31 \mathrm{ng} /$ day. Treatment with canakinumab not only induced a long-lasting complete clinical response, but also reduced the rate of IL- $1 \beta$ production to normal levels within eight weeks, suggesting that IL- $1 \beta$ production in these patients was mainly driven by IL-1 $\beta .^{30}$ This model further indicated that IL- $1 \beta$ is the only cytokine which influences disease severity and duration of response to canakinumab. It also indicated that CAPS is entirely mediated by IL-1 $\beta$, and that treatment with canakinumab restores physiological IL-1 $\beta$ production.

An open-label Phase II study in 34 CAPS patients including two with familial cold autoinflammatory syndrome, 27 with Muckle-Wells syndrome, four with severe Muckle-Wells syndrome/CINCA, and one patient with CINCA, in which canakinumab was administered either intravenously (the first four patients) or subcutaneously, showed that all but one patient achieved a complete response, with symptomatic improvement recorded within one day, and a median time to relapse of $49-115$ days. ${ }^{31}$

A 48-week, double-blind, placebo-controlled, randomized withdrawal trial was also done, consisting of a first part in which 35 patients received a single subcutaneous dose of canakinumab $(150 \mathrm{mg}$ in 31 adults and $2 \mathrm{mg} / \mathrm{kg}$ in four children weighing $<40 \mathrm{~kg}$ ). Again, all but one patient achieved a complete response by week $8 .{ }^{32}$ The remaining 34 patients were randomized to receive either placebo or subcutaneous canakinumab every eight weeks for a duration of 24 weeks. All of the patients to whom canakinumab was administered remained in remission, while disease flare occurred in 13 out of 16 patients who had received placebo. Thirty-one patients entered the third part of the study in which canakinumab was administered every eight weeks for the remainder of the 48-week study, and 28 of these patients remained in remission during this time. An increased rate of suspected infection was recorded with canakinumab versus placebo in the second part of the study ( $80 \%$ versus $56 \%$ ). Two serious adverse events were reported with canakinumab, ie, one lower urinary tract infection requiring hospitalization and one episode of vertigo, treatment of which resulted in severe headaches, and revealed closed-angle glaucoma.

Since 2008, patients who completed this trial, as well as other patients with Muckle-Wells syndrome and CINCA, have taken part in a longer-term extension trial with subcutaneous canakinumab started at $150 \mathrm{mg}$ (in patients weighing $\geq 40 \mathrm{~kg}$ ) or $2 \mathrm{mg} / \mathrm{kg}$ (patients $<40 \mathrm{~kg}$ ) every eight weeks, but with a possible adaptation of the dosage in patients who do not respond appropriately. This study involves 98 patients (including 19 with familial cold autoinflammatory syndrome, 69 with Muckle-Wells syndrome, nine with Muckle-Wells syndrome/NOMID, and one without CAPS). At an interim analysis, performed after a median duration of exposure to canakinumab of 113 days, a complete response was achieved in 41 of 44 canakinumab-naïve patients (93.2\%) at day $8 .{ }^{31}$ Interestingly, some patients required higher dosages and shorter dosing intervals, in particular low-weight children with CINCA syndrome. An improvement of quality of life was also documented. 


\section{Efficacy and safety of IL- I inhibition in other disorders}

Anakinra was initially studied in large cohorts of patients with rheumatoid arthritis. In spite of significant efficacy, this treatment failed to be as effective as other biologic agents, such as tumor necrosis factor inhibitors. On the other hand, treatment with anakinra seems to be associated with fewer serious adverse events than tumor necrosis factor inhibitors, unless used at doses $>150 \mathrm{mg} /$ day ( $50 \%$ more than the usual dosage), in particular for patients with debilitating conditions, such as diabetes. ${ }^{33}$

In patients with juvenile idiopathic arthritis, a placebocontrolled trial failed to demonstrate any significant difference between placebo and anakinra. ${ }^{34}$ However, different subsets of patients with juvenile idiopathic arthritis were included, which was almost certainly a mistake because it clearly appears that nonsystemic juvenile idiopathic arthritis on the one hand, and systemic juvenile idiopathic arthritis on the other, are very different in terms of response to biologic agents. This was illustrated some years ago by the fact that tumor necrosis factor inhibitors are much more effective in oligoarticular or polyarticular juvenile idiopathic arthritis than in systemic juvenile idiopathic arthritis. ${ }^{35}$

In systemic juvenile idiopathic arthritis, where IL-1 has been shown to play an important role, ${ }^{36,37}$ several noncontrolled studies have been published, and these suggest that most patients respond to treatment with anakinra, at least in the short term. ${ }^{36-42}$ Response rates may be higher when patients are treated at an earlier stage,,${ }^{42}$ and lower in patients with diffuse polyarthritis or arthritis involving the small joints. ${ }^{40,41}$ Very recently, the results of a multicenter, doubleblind, placebo-controlled trial in France were published..$^{43}$ In this trial, 24 patients were randomized at day 1 to receive daily subcutaneous injections of either anakinra at $2 \mathrm{mg} / \mathrm{kg}$ (maximum $100 \mathrm{mg}$ ) or placebo for one month. There was a significantly higher proportion of responders in patients randomized to anakinra (67\% versus $8 \%$ on placebo) using a set of criteria that required control of systemic symptoms, a significant decrease of both erythrocyte sedimentation rate and C-reactive protein, as well as the usual 30\% improvement in the American College of Rheumatology (ACR) core-set pediatric criteria. In the second part of the study, patients received anakinra for the total duration of the trial, ie, 12 months. Among the 22 patients who entered the second part of the study, six patients stopped anakinra treatment, in four cases because of loss of efficacy after the dose of steroids had been tapered, and in two cases because of adverse events.
The first serious adverse event was severe bowel inflammation revealing Crohn's disease at two months in a patient who was a nonresponder to anakinra after one month, and the other was an acute increase in liver enzymes at six months which resolved after anakinra was stopped. On the other hand, the antibody response to Pneumo $23^{\circledR}$ immunization (performed at day 1) was not affected by anakinra treatment. Only six patients were responders at six months, and all had inactive or nearly inactive disease. During this time, prednisone had been stopped or tapered to less than $0.3 \mathrm{mg} / \mathrm{kg}$ (or $10 \mathrm{mg} /$ day). Pharmacokinetic analyses showed that low-weight patients tended to show lower anakinra plasma concentrations. Blood gene expression profiling at enrollment and at six-month follow-up showed one set of dysregulated genes that reverted to normal values in clinical responders and a different set, including interferon-inducible genes, that was induced by IL-1 blockade.

A placebo-controlled trial was conducted with rilonacept in patients with systemic juvenile idiopathic arthritis. In this trial, patients were randomized to three small groups of 7-9 patients (two dosage levels of rilonacept were studied) and response was defined as an absence of systemic symptoms and a 30\% improvement in ACR pediatric criteria after four weeks. Preliminary results were presented. ${ }^{44}$ There was no significant difference at one month in the proportion of responders to rilonacept and placebo, but rilonacept seemed effective over the following months. Eighty-seven percent of the patients had achieved a 50\% improvement in ACR pediatric score at 12 months. ${ }^{45}$ On the other hand, $30 \%$ of patients developed injection site reactions and two patients developed macrophage activation syndrome.

A Phase II trial of canakinumab was conducted in 23 patients with systemic juvenile idiopathic arthritis to test different dosages and determine the optimal dose interval. ${ }^{46}$ Interestingly, most patients had previously failed to respond adequately to anakinra. In spite of this, canakinumab treatment, at doses ranging from 0.5 to $9 \mathrm{mg} / \mathrm{kg}$ in one or two subcutaneous injections, resulted in a $59 \%$ response rate at day 15 , with all responders having complete regression of their systemic symptomatology, a marked reduction of C-reactive protein, and a 50\% improvement in ACR coreset pediatric criteria. It is noteworthy that a majority of patients who had previously failed to respond to anakinra were responders to canakinumab. Disease flares occurred after a median delay of 56-90 days. Three patients achieved disease remission that lasted for more than one year (and two patients had still not flared at the latest follow-up) following 
one or two canakinumab injections. The steroid dose was tapered in 10 of 13 patients who were receiving steroids at baseline. In 2009, some patients who took part in the Phase II study, as well as some further patients, entered a placebocontrolled Phase III trial testing canakinumab at the dosage of $4 \mathrm{mg} / \mathrm{kg}$ (maximum $300 \mathrm{mg}$ ) every four weeks in children over three years of age.

Anti-IL-1 treatment, mainly with anakinra, has also been tested successfully in small numbers of patients with adult onset Still's disease, ${ }^{39,47,48}$ familial Mediterranean fever, ${ }^{49-51}$ tumor necrosis factor- $\alpha$ receptor-associated periodic syndrome, ${ }^{52-54}$ mevalonate kinase deficiency/hyperimmunoglobulin D syndrome), ${ }^{55-57}$ Schnitzler's syndrome, ${ }^{58-60}$ Sweet syndrome, ${ }^{61}$ Behçet's disease, ${ }^{62}$ pyogenic arthritis, pyoderma gangrenosum and acne syndrome and DIRA, ${ }^{16,63}$ relapsing polychondritis, ${ }^{64}$ and recurrent pericarditis. ${ }^{65}$ We and others have also used anakinra successfully in patients with moderate to severe myocarditis in the context of unexplained autoinflammatory syndromes. ${ }^{48}$

In gout, the NALP3 inflammasome can be activated by endogenous danger signals, ${ }^{66}$ such as monosodium urate crystals released from dying cells, and can play a role in the genesis of gouty arthritis.

Following in vitro and animal studies, a pilot study of anakinra enrolled 10 patients with acute gouty arthritis who had failed to respond to nonsteroidal anti-inflammatory drugs, colchicine, or corticosteroids. Treatment substantially relieved symptoms in all patients within 48 hours of administration of the first dose, and complete resolution of signs of arthritis was achieved in nine of the patients on day $3 .{ }^{67}$

In patients with gouty arthritis, two studies of rilonacept also demonstrated the efficacy of Il-1 blockade. In a pilot study involving 10 patients with chronic active gouty arthritis, pain scores decreased significantly during the first two weeks of treatment with rilonacept, and remained low at the end of the six-week treatment period. ${ }^{68}$ In a second study, patients starting allopurinol therapy were randomized to receive rilonacept or placebo once a week for 16 weeks. Rilonacept significantly reduced the risk of flares compared with placebo ( $22 \%$ flares with rilonacept versus $48 \%$ with placebo). ${ }^{69}$

In a dose-defining Phase II trial, patients with acute gouty arthritis refractory to and/or with contraindications to nonsteroidal anti-inflammatory drugs and/or colchicine were randomized to receive a single dose of canakinumab $10-150 \mathrm{mg}$ or the corticosteroid, triamcinolone acetonide. The pain level was assessed over the following seven days. ${ }^{70}$
All patients receiving canakinumab doses noted numerically lower pain scores than those taking triamcinolone acetonide at 72 hours postdosing, and the difference was statistically significant for the $150 \mathrm{mg}$ dose from $1-5$ days postdosing and at day 7 postdosing. At baseline, median C-reactive protein and serum amyloid A levels were greater than the upper limit of normal, but were normalized by day 7 in all canakinumab groups (except for those on the lowest dose). In contrast, in the triamcinolone acetonide group, the median C-reactive protein level remained elevated for the duration of the study. In addition, all canakinumab doses significantly reduced the risk of recurrent flare as compared with triamcinolone acetonide, with a relative risk reduction of $94 \%$. An ongoing study is investigating the efficacy of canakinumab as prophylaxis in patients initiating allopurinol. ${ }^{71}$

Case reports suggest that anakinra is an effective treatment in patients with pseudogout, where the NALP3 inflammasome is also activated. ${ }^{72,73}$ Finally, ongoing trials with IL-1 inhibitors, in particular canakinumab, may soon incorporate a broader spectrum of disorders/diseases far and beyond the already characterized autoinflammatory syndromes. ${ }^{31,74}$

\section{Conclusion}

In several models of inflammatory and, in particular, autoinflammatory, disorders such as CAPS, IL-1 blockade has shown efficacy in the short term and sometimes in the medium term. In some cases, alternative treatments exist, but IL-1 blockade is often more effective. In CAPS patients, there is no other active treatment available. Although continuously treating severe CAPS patients with IL-1 inhibitor therapy is still the only option in 2010 , the rationale for continuous treatment versus intermittent treatment is still a matter of discussion in other cases. In familial cold autoinflammatory syndrome, treatment could be limited to the coldest periods of the year for most patients. In systemic juvenile idiopathic arthritis, a subset of patients may achieve long-lasting remission after one or a few canakinumab injections. On the other hand, some rare or orphan diseases certainly warrant some effort on the part of health authorities to allow broader use of canakinumab. One problem is the cost of the drug, which is approximately $€ 12,000$ per $150 \mathrm{mg}$ vial. This cost should decrease when the indications for canakinumab expand to include other diseases, including relatively frequent disorders, such as types 1 or 2 diabetes.

When canakinumab obtained an authorization for use in CAPS, the recommended dosage in children was 
$2 \mathrm{mg} / \mathrm{kg}$ administered every eight weeks. In young low-weight children and in patients with severe Muckle-Wells syndrome or CINCA, much higher dosages and shorter dose intervals may be required. Although data regarding safety are fairly reassuring, relatively few patients have been treated with canakinumab so far; therefore there is a need for exhaustive registries to enable proper long-term follow-up of patient cohorts with different diseases. The potential for IL-1 inhibition in a range of other disorders will help us learn more about the pathogenesis of many diseases.

\section{Disclosure}

The author has previously received consulting fees from Abbott, Biovitrum, Bristol-Myers Squibb, Chugai-Roche, Merck-Serono, Novartis, Pfizer, Servier, and Wyeth. The author has also received research grants and financial support to organize educational and scientific meetings from Abbott, Amgen, Bristol-Myers Squibb, Genzyme, LFB, Novartis, Roche, Pfizer, and Wyeth.

\section{References}

1. Muckle TJ, Wells M. Urticaria, deafness, and amyloidosis: a new heredo-familial syndrome. $Q J$ Med. 1962;31:235-248.

2. Wanderer AA, Hoffman HM. The spectrum of acquired and familial cold-induced urticaria/urticaria-like syndromes. Immunol Allergy Clin North Am. 2004;24:259-286, vii.

3. Prieur AM. A recently recognised chronic inflammatory disease of early onset characterised by the triad of rash, central nervous system involvement and arthropathy. Clin Exp Rheumatol. 2001;19:103-106.

4. Hassink SG, Goldsmith DP. Neonatal onset multisystem inflammatory disease. Arthritis Rheum. 1983;26:668-673.

5. Prieur AM, Griscelli C, Lampert F, et al. A chronic, infantile, neurological, cutaneous and articular (CINCA) syndrome. A specific entity analysed in 30 patients. Scand J Rheumatol Suppl. 1987;66:57-68.

6. Cuisset L, Drenth JP, Berthelot JM, et al. Genetic linkage of the Muckle-Wells syndrome to chromosome 1q44. Am J Hum Genet. 1999;65:1054-1059.

7. Hoffman HM, Wright FA, Broide DH, Wanderer AA, Kolodner RD. Identification of a locus on chromosome 1q44 for familial cold urticaria. Am J Hum Genet. 2000;66:1693-1698.

8. Hoffman HM, Mueller JL, Broide DH, Wanderer AA, Kolodner RD. Mutation of a new gene encoding a putative pyrin-like protein causes familial cold autoinflammatory syndrome and Muckle-Wells syndrome. Nat Genet. 2001;29:301-305.

9. Feldmann J, Prieur AM, Quartier P, et al. Chronic infantile neurological cutaneous and articular syndrome is caused by mutations in CIAS1, a gene highly expressed in polymorphonuclear cells and chondrocytes. Am J Hum Genet. 2002;71:198-203.

10. Aksentijevich I, Nowak M, Mallah M, et al. De novo CIAS1 mutations, cytokine activation, and evidence for genetic heterogeneity in patients with neonatal-onset multisystem inflammatory disease (NOMID): a new member of the expanding family of pyrin-associated autoinflammatory diseases. Arthritis Rheum. 2002;46:3340-3348.

11. Martinon F, Mayor A, Tschopp J. The inflammasomes: guardians of the body. Annu Rev Immunol. 2009;27:229-265.

12. Franchi L, Eigenbrod T, Muñoz-Planillo R, Nuñez G. The inflammasome: a caspase-1-activation platform that regulates immune responses and disease pathogenesis. Nat Immunol. 2009;10: 241-247.
13. Neven B, Callebaut I, Prieur AM, et al. Molecular basis of the spectral expression of CIAS1 mutations associated with phagocytic cellmediated autoinflammatory disorders CINCA/NOMID, MWS, and FCU. Blood. 2004;103:2809-2815.

14. Dinarello CA. Interleukin-1beta and the autoinflammatory diseases. N Engl J Med. 2009;360:2467-2470.

15. Chae JJ, Wood G, Masters SL, et al. The B30.2 domain of pyrin, the familial Mediterranean fever protein, interacts directly with caspase-1 to modulate IL-1beta production. Proc Natl Acad Sci U S A. 2006;103:9982-9987.

16. Aksentijevich I, Masters SL, Ferguson PJ, et al. An autoinflammatory disease with deficiency of the interleukin-1-receptor antagonist. $N$ Engl J Med. 2009;360:2426-2437.

17. Hawkins PN, Lachmann HJ, McDermott MF. Interleukin-1receptor antagonist in the Muckle-Wells syndrome. $N$ Engl $\mathrm{J} \mathrm{Med}$. 2003;348:2583-2584.

18. Hoffman HM, Rosengren S, Boyle DL, et al. Prevention of coldassociated acute inflammation in familial cold autoinflammatory syndrome by interleukin-1 receptor antagonist. Lancet. 2004;364: 1779-1785.

19. Goldbach-Mansky R, Dailey NJ, Canna SW, et al. Neonatal-onset multisystem inflammatory disease responsive to interleukin-1beta inhibition. N Engl J Med. 2006;355:581-592.

20. Ross JB, Finlayson LA, Klotz PJ, et al. Use of anakinra (Kineret) in the treatment of familial cold autoinflammatory syndrome with a 16-month follow-up. J Cutan Med Surg. 2008;12:8-16.

21. Hawkins PN, Lachmann HJ, Aganna E, McDermott MF. Spectrum of clinical features in Muckle-Wells syndrome and response to anakinra. Arthritis Rheum. 2004;50:607-612.

22. Hawkins PN, Bybee A, Aganna E, McDermott MF. Response to anakinra in a de novo case of neonatal-onset multisystem inflammatory disease. Arthritis Rheum. 2004;50:2708-2709.

23. Frenkel J, Wulffraat NM, Kuis W. Anakinra in mutation-negative NOMID/CINCA syndrome: comment on the articles by Hawkins et al and Hoffman and Patel. Arthritis Rheum. 2004;50:3738-3739.

24. Lovell DJ, Bowyer SL, Solinger AM. Interleukin-1 blockade by anakinra improves clinical symptoms in patients with neonatalonset multisystem inflammatory disease. Arthritis Rheum. 2005;52: 1283-1286.

25. Gattorno M, Tassi S, Carta S, et al. Pattern of interleukin-1beta secretion in response to lipopolysaccharide and ATP before and after interleukin-1 blockade in patients with CIAS1 mutations. Arthritis Rheum. 2007:56:3138-3148.

26. Neven B, Marvillet I, Terrada C, et al. Long-term efficacy of the interleukin-1 receptor antagonist anakinra in ten patients with neonatalonset multisystem inflammatory disease/chronic infantile neurologic, cutaneous, articular syndrome. Arthritis Rheum. 2010;62:258-267.

27. Lepore L, Paloni G, Caorsi R, et al. Follow-up and quality of life of patients with cryopyrin-associated periodic syndromes treated with anakinra. J Pediatr. 2010;157:310-315.

28. Goldbach-Mansky R, Shroff SD, Wilson M, et al. A pilot study to evaluate the safety and efficacy of the long-acting interleukin-1 inhibitor rilonacept (interleukin-1 Trap) in patients with familial cold autoinflammatory syndrome. Arthritis Rheum. 2008;58:2432-2442.

29. Hoffman HM, Throne ML, Amar NJ, et al. Efficacy and safety of rilonacept (interleukin-1 Trap) in patients with cryopyrin-associated periodic syndromes: Results from two sequential placebo-controlled studies. Arthritis Rheum. 2008;58:2443-2452.

30. Lachmann HJ, Lowe P, Felix SD, et al. In vivo regulation of interleukin 1 beta in patients with cryopyrin-associated periodic syndromes. $J$ Exp Med. 2009;206:1029-1036.

31. Lachmann HJ, Quartier P, So A, Hawkins PN. The emerging role of interleukin-1 $\beta$ in autoinflammatory diseases. Arthritis Rheum. October 21 2010. [Epub ahead of print].

32. Lachmann HJ, Kone-Paut I, Kuemmerle-Deschner JB, et al. Use of canakinumab in the cryopyrin-associated periodic syndrome. $N$ Engl J Med. 2009;360:2416-2425. 
33. Salliot C, Dougados M, Gossec L. Risk of serious infections during rituximab, abatacept and anakinra treatments for rheumatoid arthritis: meta-analyses of randomised placebo-controlled trials. Ann Rheum Dis. 2009;68:25-32.

34. Ilowite N, Porras O, Reiff A, et al. Anakinra in the treatment of polyarticular-course juvenile rheumatoid arthritis: Safety and preliminary efficacy results of a randomized multicenter study. Clin Rheumatol. 2009;28:129-137.

35. Quartier P, Taupin P, Bourdeaut F, et al. Efficacy of etanercept for the treatment of juvenile idiopathic arthritis according to the onset type. Arthritis Rheum. 2003;48:1093-1101.

36. Pascual V, Allantaz F, Arce E, Punaro M, Banchereau J. Role of interleukin-1 (IL-1) in the pathogenesis of systemic onset juvenile idiopathic arthritis and clinical response to IL-1 blockade. J Exp Med 2005;201:1479-1486.

37. Allantaz F, Chaussabel D, Stichweh D, et al. Blood leukocyte microarrays to diagnose systemic onset juvenile idiopathic arthritis and follow the response to IL-1 blockade. J Exp Med. 2007;204 2131-2144.

38. Verbsky JW, White AJ. Effective use of the recombinant interleukin 1 receptor antagonist anakinra in therapy resistant systemic onset juvenile rheumatoid arthritis. J Rheumatol. 2004;31:2071-2075.

39. Lequerré T, Quartier P, Rosellini D, et al. Interleukin-1 receptor antagonist (anakinra) treatment in patients with systemic-onset juvenile idiopathic arthritis or adult onset Still disease: Preliminary experience in France. Ann Rheum Dis. 2008;67:302-308.

40. Gattorno M, Piccini A, Lasigliè D, et al. The pattern of response to anti-interleukin-1 treatment distinguishes two subsets of patients with systemic-onset juvenile idiopathic arthritis. Arthritis Rheum. 2008;58:1505-1515.

41. Zeft A, Hollister R, LaFleur B, et al. Anakinra for systemic juvenile arthritis: the Rocky Mountain experience. J Clin Rheumatol. 2009;15:161-164.

42. Nigrovic PA, Mannion M, Prince FH, et al. Anakinra as first-line disease modifying therapy in systemic juvenile idiopathic arthritis. Arthritis Rheum. 2010 Nov 4. [Epub ahead of print].

43. Quartier P, Allantaz F, Cimaz R, et al. A multicentre, randomised, double-blind, placebo-controlled trial with the interleukin-1 receptor antagonist anakinra in patients with systemic-onset juvenile idiopathic arthritis (ANAJIS trial). Ann Rheum Dis. December 102010. [Epub ahead of print].

44. Lovell DJ, Giannini EH, Kimura Y, et al. Preliminary evidence for sustained bioactivity of IL-1 Trap (rilonacept), a long acting IL-1 Inhibitor, in systemic juvenile idiopathic arthritis. Arthritis Rheum. 2007;56:S514.

45. Lovell DJ, Giannini EH, Kimura Y, et al. Long-term safety and efficacy of rilonacept in patients with systemic juvenile idiopathic arthritis. Presented at: European League Against Rheumatism annual scientific meeting, June 19-21 2009, Mackinac Island, MI. Abstr 2053.

46. Ruperto N, Quartier P, Wulffraat N, et al. ACZ885 (canakinumab), A new IL-1 beta blocking monoclonal antibody has a beneficial effect in children with systemic juvenile idiopathic arthritis. Presented at: European League against Rheumatism annual scientific meeting, 2009, June 10-13 2009, Copenhagen, Denmark. Abstr OP-0298.

47. Debiais S, Maillot F, Luca L, Buret J, Fautrel B, Renard JP. Efficacy of anakinra in a case of refractory Still's disease. J Clin Rheumatol. 2008;14:357-358.

48. Raffeiner B, Botsios C, Dinarello C, Ometto F, Punzi L, Ramonda R. Adult-onset Still's disease with myocarditis successfully treated with the interleukin-1 receptor antagonist anakinra. Joint Bone Spine. October 29 2010. [Epub ahead of print].

49. Mitroulis I, Papadopoulos VP, Konstantinidis T, Ritis K. Anakinra suppresses familial Mediterranean fever crises in a colchicine-resistant patient. Neth J Med. 2008;66:489-491.

50. Moser C, Pohl G, Haslinger I, et al. Successful treatment of familial Mediterranean fever with anakinra and outcome after renal transplantation. Nephrol Dial Transplant. 2009;24:676-678.
51. Petropoulou AD, Robin M, Socié G, Galicier L. Transmission of familial Mediterranean fever mutation after bone marrow transplantation and successful treatment with anakinra. Transplantation. 2010;90:102-103.

52. Sacré K, Brihaye B, Lidove O, et al. Dramatic improvement following interleukin 1 beta blockade in tumor necrosis factor receptor-1associated syndrome (TRAPS) resistant to anti-TNF-alpha therapy. J Rheumatol. 2008;35:357-358.

53. Gattorno M, Pelagatti MA, Meini A, et al. Persistent efficacy of anakinra in patients with tumor necrosis factor receptor-associated periodic syndrome. Arthritis Rheum. 2008;58:1516-1520.

54. Obici L, Meini A, Cattalini M, et al. Favourable and sustained response to anakinra in tumour necrosis factor receptor-associated periodic syndrome (TRAPS) with or without AA amyloidosis. Ann Rheum Dis. December 20 2010. [Epub ahead of print].

55. Lequerré T, Vittecoq O, Pouplin S, et al. Mevalonate kinase deficiency syndrome with structural damage responsive to anakinra. Rheumatology (Oxford). 2007;46:1860-1862.

56. Van der Hilst JC, Bodar EJ, Barron KS, et al. Long-term follow-up, clinical features, and quality of life in a series of 103 patients with hyperimmunoglobulinemia D syndrome. Medicine (Baltimore). 2008;87:301-310

57. Van der Hilst JC, Frenkel J. Hyperimmunoglobulin D syndrome in childhood. Curr Rheumatol Rep. 2010;12:101-107.

58. Schuster C, Kränke B, Aberer E, Arbab E, Sturm G, Aberer W. Schnitzler syndrome: response to anakinra in two cases and a review of the literature. Int J Dermatol. 2009;48:1190-1194.

59. Cascavilla N, Bisceglia M, D’Arena G. Successful treatment of Schnitzler's syndrome with anakinra after failure of rituximab trial. Int J Immunopathol Pharmacol. 2010;23:633-636.

60. Besada E, Nossent H. Dramatic response to IL1-RA treatment in longstanding multidrug resistant Schnitzler's syndrome: a case report and literature review. Clin Rheumatol. 2010;29:567-571.

61. Delluc A, Limal N, Puéchal X, Francès C, Piette JC, Cacoub P. Efficacy of anakinra, an IL1 receptor antagonist, in refractory Sweet syndrome. Ann Rheum Dis. 2008;67:278-279.

62. Botsios C, Sfriso P, Furlan A, Punzi L, Dinarello CA. Resistant Behçet disease responsive to anakinra. Ann Intern Med. 2008;149:284-286.

63. Brenner M, Ruzicka T, Plewig G, Thomas P, Herzer P. Targeted treatment of pyoderma gangrenosum in PAPA (pyogenic arthritis, pyoderma gangrenosum and acne) syndrome with the recombinant human interleukin-1 receptor antagonist anakinra. $\mathrm{Br} J$ Dermatol. 2009;161:1199-1201.

64. Wendling D, Govindaraju S, Prati C, Toussirot E, Bertolini E. Efficacy of anakinra in a patient with refractory relapsing polychondritis. Joint Bone Spine. 2008;75:622-624.

65. Picco P, Brisca G, Traverso F, Loy A, Gattorno M, Martini A. Successful treatment of idiopathic recurrent pericarditis in children with interleukin-1beta receptor antagonist (anakinra): an unrecognized autoinflammatory disease? Arthritis Rheum. 2009;60: 264-268.

66. Martinon F, Petrilli V, Mayor A, Tardivel A, Tschopp J. Gout-associated uric acid crystals activate the NALP3 inflammasome. Nature. 2006;440:237-241.

67. Neogi T. Interleukin-1 antagonism in acute gout: is targeting a single cytokine the answer? Arthritis Rheum. 2010;62:2845-2849.

68. Schumacher RH, Sundy JS, Terkeltaub R, et al. Placebo-controlled study of rilonacept for prevention of gout flares during initiation of urate-lowering therapy. Ann Rheum Dis. 2009;68 Suppl 3:680.

69. Terkeltaub R, Sundy JS, Schumacher HR, et al. The interleukin 1 inhibitor rilonacept in treatment of chronic gouty arthritis: results of a placebo-controlled, monosequence crossover, non-randomised, singleblind pilot study. Ann Rheum Dis. 2009;68:1613-1617.

70. So A, de Meulemeester M, Pikhlak A, et al. Canakinumab for the treatment of acute flares in difficult-to-treat gouty arthritis: results of a multicenter, phase II, dose-ranging study. Arthritis Rheum. 2010;62:3064-3076. 
71. Schlesinger N, Lin HY, de Meulemeester M, et al. Efficacy of canakinumab (ACZ885) in the prevention of flares in gout patients initiating allopurinol therapy. Ann Rheum Dis. 2010;69 Suppl 3:121.

72. McGonagle D, Tan AL, Madden J, Emery P, McDermott MF. Successful treatment of resistant pseudogout with anakinra. Arthritis Rheum. 2008;58:631-633.
73. Announ N, Palmer G, Guerne PA, Gabay C. Anakinra is a possible alternative in the treatment and prevention of acute attacks of pseudogout in end-stage renal failure. Joint Bone Spine. 2009;76:424-426.

74. Mitroulis I, Skendros P, Ritis K. Targeting IL-1beta in disease; the expanding role of NLRP3 inflammasome. Eur J Intern Med. 2010;2:157-163.

\section{Publish your work in this journal}

Open Access Rheumatology Research and Reviews is an international, peer-reviewed, open access journal, publishing all aspects of clinical and experimental rheumatology in the clinic and laboratory including the following topics: Pathology, pathophysiology of rheumatological diseases; Investigation, treatment and management of rheumatological diseases; Clinical trials and novel pharmacological approaches for the treatment of rheumatological disorders. The manuscript management system is completely online and includes a very quick and fair peerreview system, which is all easy to use. Visit http://www.dovepress.com/ testimonials.php to read real quotes from published authors. 